\title{
Mechanical Properties of Tetragonal and Orthorhombic Phases of Quasi-One-Dimensional Antiferromagnet $\mathrm{KCuF}_{3}$
}

\author{
D. LEGUT ${ }^{a, *}$ U.D. WDOWIK ${ }^{b}$ \\ ${ }^{a}$ Nanotechnology Centre, VSB-Technical University of Ostrava, 17. listopadu 15, 70833 Ostrava, Czech Republic \\ ${ }^{b}$ Institute of Technology, Pedagogical University, Podchorążych 2, 30-084 Kraków, Poland
}

\begin{abstract}
The density functional theory was used to calculate the elastic constants for the two tetragonal (a-type) and (d-type), and the orthorhombic structures of $\mathrm{KCuF}_{3}$. Based on the single elastic constants the polycrystalline mechanical properties such as bulk, shear, Young moduli and Poisson ratio using Reuss-Voigt-Hill averaging method were estimated. Furthermore, the sound transverse and longitudional velocities (along the $a$-axis and $c$-axis) were calculated and the Debye temperatures were determined for all three investigated phases.
\end{abstract}

DOI: 10.12693/APhysPolA.126.14

PACS: 62.20.fk, 73.61.Ng, 62.20.de, 62.20.dj, 75.10.Pq

\section{Introduction}

$\mathrm{KCuF}_{3}$ is well-known example of the one-dimensional magnetic system in three-dimensional magnetic ion sublattice [1] that exists in three different structures; two of them possessing tetragonal symmetry $(I 4 / \mathrm{mcm}$ and $P 4 / \mathrm{mbm}$ ) and one with orthorhombic symmetry $\left(P 2_{1} 2_{1} 2_{1}\right)$ [1-2]. For the extended list of physical quantities known about the $\mathrm{KCuF}_{3}$ as well as the description of the lattice dimensions and Wyckoff positions of the constituents we refer the reader to the recent publication [3]. Here, we report the single crystal elastic constants of the all three polymorphs of $\mathrm{KCuF}_{3}$ using ab initio calculations. Based on those, we investigate the mechanical properties, such as Young, bulk and shear moduli, Poisson ratios, ductility and the Debye temperatures using longitudional and transverse sound velocities.

\section{Methodology}

First-principles calculations were performed using the density functional theory [4] in a single electron framework of the VASP code [5]. For details we refer the reader to Ref. [3] where all parameters are specified. For the calculations of elastic constants $\left(C_{i j}\right)$ we applied methodology implemented in the VASP code.

\section{Results and discussion}

The elastic constants of both tetragonal and orthorhombic $\mathrm{KCuF}_{3}$ structures are summarized in Table I. In general, the intrinsic mechanical stability of a solid is determined by certain conditions concerning the values of $C_{i j}$ related to the crystal symmetry [6]. For all $\mathrm{KCuF}_{3}$ polymorphs the $C_{i j}$ are positive. The stability conditions

*corresponding author; e-mail: dominik. legut@vsb.cz for tetragonal $(I 4 / \mathrm{mcm}$ and $P 4 / \mathrm{mbm})$ and orthorhombic $\left(P 2_{1} 2_{1} 2_{1}\right)$ polymorphs, see e.g. [7], are here fulfilled and hence these three phases of $\mathrm{KCuF}_{3}$ are mechanically stable. The slight differences between $C_{i j}$ of the two tetragonal polymorphs are observed predomintalty for $C_{11}, C_{12}$, and $C_{13}$, all being higher (stiffer) by about $4-7 \mathrm{GPa}$ in the $P 4 / \mathrm{mbm}$ structure with respect to the $I 4 / \mathrm{mcm}$ structure, whereas the $C_{33}, C_{44}$, and $C_{66}$ are only slightly higher. The increase in $C_{i j}$, can be attributed to the different location of the fluorine atoms within the tetragonal plane, see e.g. Fig. 1 in [3]. In general, the structure with orthorhombic symmetry is described by 9 independent elastic constants. Results of calculations shown in Table I indicate that the $P 2_{1} 2_{1} 2_{1}$ structure of $\mathrm{KCuF}_{3}$ behaves elastically as being almost tetragonal one, having the values of $C_{11} \approx C_{22}, C_{13} \approx C_{23}$, and $C_{44} \approx C_{55}$.

TABLE I

Elastic constants $C_{i j}[\mathrm{GPa}]$ of tetragonal $(I 4 / \mathrm{mcm}$, $P 4 / \mathrm{mbm}$ ) and orthorhombic polymorphs of $\mathrm{KCuF}_{3}$.

\begin{tabular}{c|c|c|c|c|c|c|c|c|c}
\hline \hline polymorph & $C_{11}$ & $C_{12}$ & $C_{13}$ & $C_{22}$ & $C_{23}$ & $C_{33}$ & $C_{44}$ & $C_{55}$ & $C_{66}$ \\
\hline$I 4 / \mathrm{mcm}$ & 70 & 18 & 33 & - & - & 129 & 29 & - & 25 \\
\hline$P 4 / m b m$ & 77 & 23 & 37 & - & - & 130 & 31 & - & 28 \\
\hline$P 2_{1} 2_{1} 2_{1}$ & 65 & 25 & 35 & 66 & 36 & 134 & 29.9 & 30.3 & 28
\end{tabular}

For low symmetry phases the isotropic polycrystalline elastic constants, bulk $\left(B_{R V H}\right)$ and shear $\left(G_{R V H}\right)$ moduli can be calculated by applying the so-called ReussVoigt-Hill averaging method and using the single elastic constants $C_{i j}[8,9]$. The mechanical properties of the polycrystalline solid such are Young modulus $(E)$, Poisson ratio $(\nu)$, and $B_{R V H} / G_{R V H}$ (Pugh's modulus ratio [10]) are also determined and listed in Table II. The Pugh's modulus ratio is usually used as an empirical rule to distinguish the ductile $\left(B_{R V H} / G_{R V H}>1.75\right)$ from the brittle material $\left(B_{R V H} / G_{R V H}<1.75\right)$. One can see in Table II that the orthorhombic phase is on the edge of being brittle, whereas both tetragonal phases are rather ductile. Similarly, the ductility is correlated with the lower values of $E$ and $G_{R V H}$ and higher value of $\nu$, i.e. 
with weaker and less directional bonding [11]. Hence, corresponding values of Table II indicate that the ductility decreases going from $I 4$ to $P 4$ and to $P 2_{1} 2_{1} 2_{1}$ structure of $\mathrm{KCuF}_{3}$.

\section{TABLE II}

The Reuss-Voigt-Hill averaged bulk $\left(B_{R V H}\right)$, shear $\left(G_{R V H}\right)$ and Young $(E)$ moduli in GPa, Pugh's modulus ratio $\left(B_{R V H} / G_{R V H}\right)$, Poisson ratio $(\nu)$ of tetragonal $(I 4 / \mathrm{mcm}, P 4 / \mathrm{mbm})$ and orthorhombic $\left(P 2_{1} 2_{1} 2_{1}\right)$ polymorphs of $\mathrm{KCuF}_{3}$.

\begin{tabular}{c|c|c|c|c|c}
\hline \hline polymorph & $B_{R V H}$ & $G_{R V H}$ & $B_{R V H} / G_{R V H}$ & $E$ & $\nu$ \\
\hline$I 4 / m c m$ & 45 & 23 & 1.99 & 59 & 0.285 \\
\hline$P 4 / m b m$ & 51 & 24 & 2.13 & 62 & 0.294 \\
\hline$P 2_{1} 2_{1} 2_{1}$ & 48 & 28 & 1.71 & 70 & 0.254
\end{tabular}

Next, we determine the Debye temperature using longitudional $\left(v_{l}\right)$ and transverse $\left(v_{t}\right)$ sound velocities obtained from $B_{R V H}$ and $G_{R V H}$ following the Ref. [12-14]. The mean sound velocity $\left(v_{m}\right)$, used for the estimation of Debye temperature, was obtained as $\nu_{m}=\nu_{l} \frac{2 s^{2}+1}{2 s^{3}+1}$, where $s=\frac{\nu_{l}}{\nu_{t}}$. All three phases have very similar volume per formula unit [3] leading to the density of $3.64 \mathrm{~g} / \mathrm{cm}^{3}$, whereas the experimental value is $3.94 \mathrm{~g} / \mathrm{cm}^{3}$ [13]. The former value is used to evaluate the sound velocities. Results are summarized in Table III. For a comparison, we also derived the Debye temperature from the phonon density of states $\left(\theta_{D p h}\right)$, published in Ref. [3], using the procedure described in Ref. [15]. For all three phases the $\theta_{D}$ (based on $C_{i j}$ ) are in very good agreement with $\theta_{D p h}$ obtained from the atomic vibrations (phonons). The effect of the experimental $\rho$ on $\theta_{D}$ is negligible, as it lowers $\theta_{D}$ by less than $5 \mathrm{~K}$.

\section{TABLE III}

The longitudional, transverse and mean sound velocities and the Debye temperature calculated using $\mathrm{C}_{i j}\left(\theta_{D}\right)$ and from the phonon density of states $\theta_{D p h}$ of Ref. [3].

\begin{tabular}{c|c|c|c|c|c}
\hline \hline phase & $v_{l}(\mathrm{~cm} / \mathrm{s})$ & $v_{t}(\mathrm{~cm} / \mathrm{s})$ & $v_{m}(\mathrm{~cm} / \mathrm{s})$ & $\theta_{D}(\mathrm{~K})$ & $\theta_{D p h}(\mathrm{~K})$ \\
\hline$I 4 / m c m$ & $4.578+\mathrm{E} 5$ & $2.512+\mathrm{E} 5$ & $2.800+\mathrm{E} 5$ & 261 & 276 \\
\hline$P 4 / m b m$ & $4.775+\mathrm{E} 5$ & $2.577+\mathrm{E} 5$ & $2.877+\mathrm{E} 5$ & 268 & 292 \\
\hline$P 2_{1} 2_{1} 2_{1}$ & $4.827+\mathrm{E} 5$ & $2.772+\mathrm{E} 5$ & $3.079+\mathrm{E} 5$ & 289 & 294
\end{tabular}

It seems that the increase of the Debye temperature $\left(\theta_{D}\right)$ and decrease of the ductility correlates with the phase stability of the $\mathrm{KCuF}_{3}$ polymorphs. The lowest $\theta_{D}$ and higher ductility have tetragonal $I 4 / \mathrm{mcm}$ and $P 4 / \mathrm{mcm}$ structures whereas the brittle behavior possesses the least stable orthorhombic $P 2_{1} 2_{1} 2_{1}$ structure.

\section{Conclusions}

Using the density functional theory calculations we determined the mechanical properties of two tetragonal and one orthorhombic phase of the quasi-one-dimensional antiferromagnet $\mathrm{KCuF}_{3}$. Using Hill averaged calculated single elastic constants $\left(C_{i j}\right)$ we showed that the brittle phase is the orthorhombic one, whereas both tetragonal phases are rather ductile. We estimated Debye temperatures for all three phases using $C_{i j}$ and phonon density of states of Ref. [3], all values being in very good accord.

\section{Acknowledgments}

Interdisciplinary Centre for Mathematical and Computational Modelling (ICM), Warsaw University, Poland and National Center CERIT Scientific Cloud (CERITSC), Czech Republic are acknowledged for providing the computer facilities to support part of the present calculations under Grants No. G28-12 and Reg. No. CZ.1.05/3.2.00/08.0144. D. Legut acknowledges a support within the framework of the Nanotechnology Centre - Nanotechnology - the basis for international cooperation project, Reg. No. CZ.1.07/2.3.00/20.0074 and the IT4-Innovations Centre of Excellence project, Reg. No. CZ.1.05/1.1.00/02.0070, both supported by Operational Programme 'Education for competitiveness' funded by Structural Funds of the European Union and state budget of the Czech Republic.

\section{References}

[1] A. Okazaki, Y. Suemune, J. Phys. Soc. Japan 16, 671 (1961).

[2] M. Hidaka, T. Eguchi, I. Yamada, J. Phys. Soc. Japan 67, 2488 (1998).

[3] D. Legut, U.D. Wdowik, J. Phys.: Condens. Matter 25, 115404 (2013).

[4] P. Hohenberg, W. Kohn, Phys. Rev. 136, B864 (1964).

[5] G. Kresse, J. Furthmueller, Phys. Rev. B 54, 11169 (1996).

[6] D.C. Wallace, Thermodynamics of Crystals, Willey, New York 1972

[7] O. Beckstein, J.E. Klepeis, G.L.W. Hart, O. Pankratov, Phys. Rev. B 63, 134112 (2001).

[8] R. Hill, Proc. Phys. Soc. A 65, 349 (1952).

[9] G. Grimvall, Thermophysical Properties of Materials Elsevier, Amsterdam 1999. ISBN-10: 0444827943.

[10] S.F. Pugh, Phil. Mag. 45, 823 (1954).

[11] F. Chu, T.E. Mitchell, B. Majumdar, D. Miracle, T.K. Nandy, D. Banerjee, Intermetallics 5, 147 (1997).

[12] O.L. Anderson, J. Phys. Chem. Solids 24, 909 (1963).

[13] R. Berman, Thermal Conductivity in Solids Clarendon Press, Oxford 1976. ISBN: 0198514298.

[14] V. Tkáč, A. Orendáčová, M. Orendáč, D. Legut, K. Tibenská, A. Feher, M. Poirier, M.W. Meisel, Acta Phys. Pol. A 121, 1098 (2012).

[15] D. Legut, U.D. Wdowik, J. Phys. Cond. Matter. 22, 435402 (2010). 